\title{
ASSOCIAÇÃO ENTRE OS EVENTOS DE ENOS E A DISTRIBUIÇÃO ESPAÇO- TEMPORAL DA PRECIPITAÇÃO NA BACIA HIDROGRÁFICA DO RIO CAPIM (MA-PA)
}

\author{
DIAS, Gustavo Francesco de Morais - gustavo.dias@ifpa.edu.br \\ Instituto Federal do Pará / IFPA
GOMES, Dênis José Cardoso - deniss.feg@gmail.com Universidade Federal do Pará / UFPA
SERRÃO, Edivaldo Afonso Oliveira - oliveiraserrao@gmail.com Universidade Federal de Campina Grande / UFCG
LIMA, Aline Maria Meiguins de - alinemeiguins@gmail.com Universidade Federal do Pará / UFPA
MIRANDA, Sarah Brasil de Araújo de - sarahbrasildam@gmail.com Universidade Federal Rural da Amazônia / UFRA
SILVA JUNIOR, João de Athaydes - athaydes@ufpa.br Universidade Federal do Pará / UFPA
SOUSA, Adriano Marlisom Leão de - marlisomleao@yahoo.com.br Universidade Federal Rural da Amazônia/ UFRA

\begin{abstract}
RESUMO: A bacia hidrográfica do rio Capim está localizada no nordeste do estado do Pará, compondo o eixo de maior circulação econômica estadual direcionado ao longo da rodovia Belém-Brasília. O estudo da variabilidade espaço-temporal da precipitação relacionadas á eventos climáticos são muito importantes para determinar a sua influência no ciclo hidrológico, na agricultura, geração de energia, além da caracterização do regime hídrico da bacia hidrográfica, tornando-se fundamental para o gerenciamento dos recursos hídricos na mesma. O presente trabalho tem como objetivo analisar a distribuição espacial e temporal da precipitação na bacia hidrográfica do rio Capim, no período de 1983 a 2014, definindo-se as regiões de maior e menor potencial hídrico. Utilizou-se os dados de reanálise de precipitação do GPCC, devido à baixa densidade de pluviômetros na região. Para validação dos dados foram feitos cálculos estatísticos ( $R$, $\mathrm{R}^{2}$, N-S, IC e U) entre os dados do GPCC e da ANA. As anomalias pluviométricas foram obtidas através do cálculo do desvio padrão. Os resultados apontam que por meio da espacialização da precipitação foram observados maiores valores para a região do baixomédio Capim e menores valores para o alto Capim. Além da identificação de uma estação chuvosa com início em dezembro e término em maio que estão associadas a padrões climáticos do acoplamento oceano-atmosfera.
\end{abstract}

PALAVRAS-CHAVE: Precipitação; Bacia hidrográfica; Eventos climáticos

ASSOCIATION BETWEEN ENOSE EVENTS AND THE SPACE-TEMPORAL DISTRIBUTION OF PRECIPITATION IN THE CAPIM RIVER HYDROGRAPHIC BOWL

ABSTRACT: The catchment area of the Capim River is located in the northeast of the State of Pará, forming the axis of greater economic circulation of the State directed along the Belém-Brasília Highway. The total watercourse is $967 \mathrm{~km}$ and 1,500 meters of mouth, not having its own spring. It arises from the confluence of two great rivers that are the Ararandeua and the Surubiju to the northwest of the mountain range of the Gurupi, to 103 meters of height. The study of the use of hydrological processes relating them to the use and coverage of the soil in the limits of the basin is fundamental for the 
decision making and planning of the management of the water resources. The present work has the objective of analyzing the spatial distribution of precipitation in the catchment area of the Capim river, from 1983 to 2014, defining the regions with higher and lower water potential. The results indicate that by means of the precipitation spatialization, higher values were observed for the low-medium Capim region and lower values for the Upper Capim. In addition to identifying a rainy season beginning in December and ending in may that are associated with weather patterns of the oceanatmosphere coupling.

KEYWORDS: Precipitation; River basin; Climate events.

\section{INTRODUÇÃO}

Atualmente a hidrologia aplicada está voltada para os diferentes estudos acerca da utilização dos recursos hídricos, como a preservação do meio ambiente e mudanças no uso das terras em bacias de drenagem. Neste sentido, as análises da precipitação em bacias hidrográficas são fundamentais para o conhecimento dos processos hidrológicos regionais (SILVA, 2014).

Segundo Bertoni e Tucci (2009), a precipitação é toda água proveniente do meio atmosférico que atinge a superfície terrestre em suas diferentes formas como: neblina, chuva, granizo, saraiva, orvalho, geada e neve. Dentre estas, a chuva é considerada o tipo de precipitação mais importante para o estudo da hidrologia, devido ao seu escoamento. A precipitação que atinge o solo promove o escoamento superficial, infiltração no solo e reter-se em depressões, sendo esses processos dependentes do tipo de solo e cobertura da terra (CARAM, 2010).

Segundo Amanajás e Braga (2012), a precipitação não é homogênea sobre a superfície terrestre, pois fatores regionais e locais como a topografia e direção do vento influenciam na distribuição espaço-temporal da chuva nas diversas regiões do planeta. Em seu estudo para identificar regiões homogêneas de precipitação na Amazônia oriental, verificaram a existência de 4 regiões com padrões pluviométricos distintos, quanto à variabilidade sazonal e interanual da precipitação.

O estudo da distribuição espaço-temporal pluviométrico são muito importantes para determinar a sua influência no ciclo hidrológico, na agricultura, geração de energia, dentre outras atividades. Além disso, esse estudo é imprescindível para a caracterização do regime hídrico em uma dada bacia hidrográfica, tornando-se fundamental para o gerenciamento dos recursos hídricos na mesma (WANDERLEY et al., 2012).

De acordo com Gomes (2008) a utilização do GPCC é essencial, pois a interpolação de dados dos postos de pluviômetros compensa a falta de informação hidrológica na Amazônia oriental devido a distribuição irregular das estações pluviométricas (NASCIMENTO et al., 2010).

A maior parte da precipitação anual da Amazônia ocorre entre as estações de verão e outono, entre os meses de dezembro a maio, devido aos padrões de circulação atmosférica de grande escala associados à Zona de Convergência do Atlântico Sul (ZCAS) e a Zona de Convergência Intertropical (ZCIT) (SOUZA et al., 2009; ÁVILA et al., 2014).

O fenômeno El Niño-Oscilação Sul (ENOS) é um fenômeno de interação oceano-atmosfera, que ocorre no oceano Pacífico Equatorial, sendo considerado como a principal causa da variabilidade climática em diversas regiões do globo. 
Esse fenômeno apresenta duas fases extremas: uma fase quente, denominada El Niño, e uma fase fria, a La Niña (ANDREOLI; KAYANO, 2005). Na Amazônia os eventos extremos de El Niño (La Niña) estão associados ao aquecimento (resfriamento) superficial das águas do Pacífico Equatorial. Essas alterações do comportamento climático, atingem a região norte da Amazônia, podendo acarretar em secas e cheias, influenciando as estações da região (MARCUZZO; ROMERO, 2013).

Marengo (1992) sugere que a diminuição da chuva no norte da Amazônia, região na qual o efeito do ENOS é mais intenso, coincide com eventos de El Niño fortes, como os que ocorreram em 1983 e 1987. Segundo Sousa et al. (2015), a variação espacial média anual da precipitação na Amazônia quando associada a extremos climáticos implica na diminuição da precipitação de oeste para leste.

No estudo realizado por Braga (2001), identificou-se que a intensidade das variações relacionadas a precipitação e a vazão dos rios na bacia do GuamáCapim (Nordeste Paraense) são diferentes nas suas regiões de norte a sul. Isso ocorre pelo fato do evento El Niño (La Niña) diminuir (aumentar) a convecção na bacia ocasionando a diminuição (aumento) do regime pluviométrico (SOUSA et al., 2015).

A variabilidade nos totais pluviométricos regionais possibilitam uma maior vulnerabilidade da população a tal comportamento climático e suas variabilidades, logo, estudos envolvendo as causas e consequências das variações das chuvas são imprescindíveis para gerar informações e produtos climatológicos úteis ao benefício da sociedade (SOUZA et al., 2009).

Tais fatores, tornam o diagnóstico do balanço hídrico vulnerável às várias modificações que as formas de uso e cobertura da terra podem induzir, logo a precipitação pluviométrica passa a ter maior contribuição para o abastecimento hídrico, tornando assim o sistema susceptível à ocorrência de eventos extremos do clima. Desta forma, buscou-se discutir o comportamento da precipitação pluviométrica em uma bacia da Amazônia Oriental (considerando o período de 1983 a 2014), a bacia do rio Capim, que exemplifica uma região de demanda crescente pelo uso de água e econômico do território, mas com baixa densidade de informação pluviométrica, fluviométrica e vulnerável aos efeitos da ocorrência de eventos extremos associados aos períodos de ENOS na região.

\section{MATERIAL E MÉTODOS}

\section{ÁREA DE ESTUDO}

A bacia do rio Capim está localizada no nordeste do estado do Pará (Figura 1), é definido como clima AF segundo classificação climática de köppengeiger, com temperatura média de $30^{\circ} \mathrm{C}$ e precipitação anual entorno de 2050 $\mathrm{mm}$, compondo o eixo de maior circulação econômica do Estado direcionado ao longo da Rodovia Belém-Brasília (BR 010). 


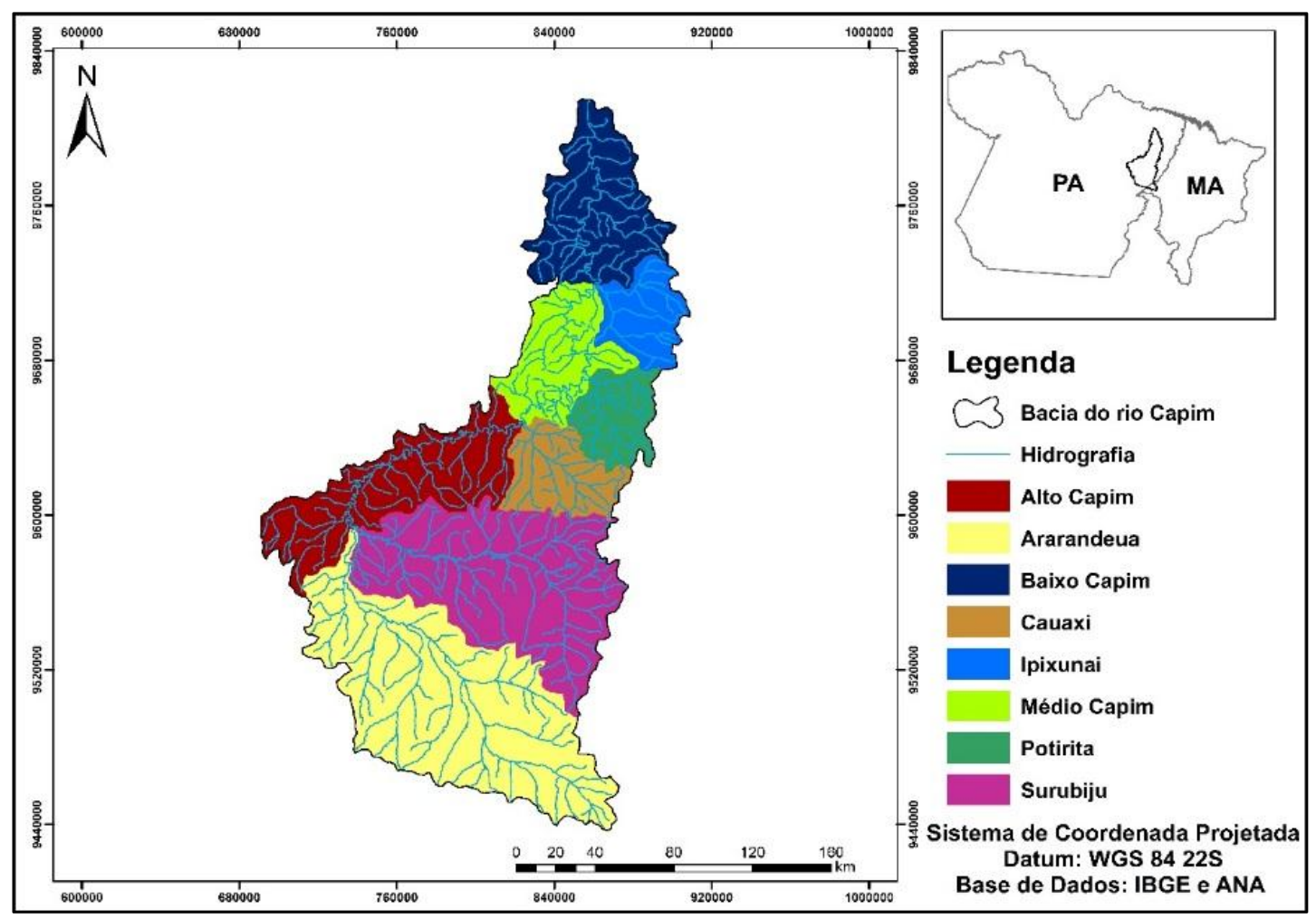

Figura 1 - Bacia do rio Capim na parte brasileira da bacia hidrográfica amazônica e as divisões de suas sub-bacias.

Destaca-se por concentrar ações de infraestrutura voltadas ao desenvolvimento econômico local, tais como: a hidrovia Guamá-Capim, os projetos industriais e mineiros e a expansão das atividades extrativista e agropecuárias, nos municípios da região (LIMA; PONTE, 2012).

A bacia do rio Capim apresenta uma área total de $37.485,75 \mathrm{~km}^{2}$, da qual $95,22 \%$ pertence ao Pará e $4,78 \%$ ao Maranhão (LIMA, 2007). Adotou-se no estudo os limites definidos pelo Sistema Ottobacias (Resolução n. 30/2002 do Conselho Nacional de Recursos Hídricos), na "Ordem 4". No qual a bacia do rio Capim apresenta 8 sub-bacias, entre elas estão a do Baixo Capim, Médio Capim, Alto Capim, Potirita, Cauaxi, Ipixunai, Ararandeua e Surubiju.

\section{DADOS E MÉTODOS}

\section{CARACTERIZAÇÃO PLUVIOMÉTRICA DA BACIA DO RIO CAPIM}

Devido à baixa densidade de estações pluviométricas com dados consistentes para a bacia de estudo, optou-se por utilizar os dados de reanálise de precipitação do Precipitation Climatology Centre (GPCC), através do Earth System Research Laboratory's (ESRL) e Physical Sciences Division (PSD) da NOAA, com grade de $\left(1^{\circ} \times 1^{\circ}\right)$ de resolução espacial. Os dados de reanálise do GPCC são um produto de interpolação de 7.000 estações com dados de precipitação ao redor do mundo. Foram selecionados os dados mensais de 1983 a 2014 referentes ao quadrante que cobre todo o limite da bacia do rio Capim, o 
qual compreende as latitudes entre $6^{\circ} \mathrm{S}$ a $1^{\circ} \mathrm{S}$ e as longitudes entre $50^{\circ} \mathrm{W}$ a 460W.

Os dados do GPCC já foram testados e validados por diverços autores como (SANTOS, et al., 2014; SCHNEIDER et al., 2016; Schneider et al., 2017), porém, no intuito de averiguar se os dados do GPCC estão coerentes com os dados observados na bacia do capim, foi feito a validação dos dados do GPCC com os dados observados pela ANA, da estação de Rondon do Pará codigo 448000. Disponibilizados a través banco de dados de série histórica. Pra isso foi utilizado as equações ( 1 a 5 ) descritas a seguir, seguindo a metodologia de (Guilhon e Rocha, 2007 ; Serrão et al., 2017).

O $r^{2}$ (equação 1 ) é definido como a relação que mede a proporção da variação total da variável dependente que é explicada pela variação da variável independente.

$$
R^{2}=\frac{\sum_{i=1}^{n}\left(\widehat{Y_{I}}-\bar{Y}\right)^{2}}{\sum_{i=1}^{n}\left(Y_{I}-\bar{Y}\right)^{2}}
$$

Sendo: $\sum_{i=1}^{n}\left(\widehat{Y}_{I}-\bar{Y}\right)^{2}$ corresponde à variação explicada, e $\sum_{i=1}^{n}\left(Y_{I}-\bar{Y}\right)^{2}$ é a variação não explicada.

O coeficiente de correlação de Pearson (r) (equação 2). Este coeficiente assume valores entre -1 e 1 . Quando 1 significa correlação perfeita positiva entre as duas variáveis, quando -1 significa correlação negativa perfeita entre as duas variáveis, e quando 0 significa que as duas variáveis não dependem linearmente uma da outra.

$$
R=\frac{\sum(X-\bar{X}) \cdot(Y-\bar{Y})}{\sqrt{\sum(X-\bar{X})^{2} \cdot \sum(Y-\bar{Y})^{2}}}
$$

O coeficiente de eficiência de Nash-Sutcliffe-NS inicialmente utilizado para avaliar o desempenho de modelos hidrológicos (Nash; Sutcliffe, 1970), também eficiente na calibração de estimada de precipitação por satélite e precipitação observada (Guilhon; Rocha, 2007). Pode variar entre negativo infinito a 1 , sendo o valor 1 indicativo de um perfeito ajuste. Para este estudo considerou-se como excelentes valores de NS acima de 0,90 em que: $\bar{P}_{O B S}$, é a precipitação média mensal de acordo com os dados observados do INMET e $\bar{P}_{E S T}$ é a precipitação estimada pelo GPCC.

$$
N S=1-\frac{\sum_{i=1}^{n}\left(P_{E S T}-P_{O B S}\right)^{2}}{\sum_{i=1}^{n}\left(P_{O B S}-\bar{P}_{O B S}\right)^{2}}
$$

O índice de concordância de Willmott (IC) mede o grau em que a precipitação estimada se aproxima da precipitação observada na estação. Este índice varia de zero a um, sendo zero, nenhuma concordância e um, concordância perfeita e é dado por: 


$$
I C=1-\frac{\sum_{i=1}^{n}\left(V_{E S T}-V_{O B S}\right)^{2}}{\sum_{i=1}^{n}\left(\left|V_{E S T}-\bar{V}_{O B S}\right|+\left|V_{O B S}-\bar{V}_{O B S}\right|\right)^{2}}
$$

O coeficiente de $U$ (Theil) (equação 5), consiste em uma medida qualitativa de precisão, ao compara os dados estimados com os dados observados (Spencer, 1993). Assim quando: $U>1$, o erro da estimativa é maior que o observado, superestimando o real. $U<1$, o erro da estimativa é menor que o observado, subestimando o real. $U \cong 0$, melhor será o resultado dos dados estimados em relação ao medido.

$U=\sqrt{\frac{\sum_{i=1}^{n}\left(V_{S W A T}-V_{o b s}\right)^{2}}{\left(\sum_{i=1}^{n}\left(V_{o b s}\right)^{2}\right.}}$

Para o estudo temporal da precipitação na bacia do rio Capim foram produzidos gráficos a partir do Software $R$, com auxílio do Software R Studio, com as médias das precipitações mensais e anuais para o período em estudo. A fim de analisar a distribuição espacial da precipitação na bacia do rio Capim, foram construídos mapas utilizando a técnica de interpolação de krigagem. Segundo Lundgren et al. (2016) no software ArcGis, versão para estudantes, a krigagem permite estimar o valor de um atributo em um local qualquer, apenas tomando o valor do atributo em alguns locais conhecidos e próximos ao local em que o atributo será estimado. A krigagem é um método geoestatístico utilizado para interpolação, o qual se baseia na teoria das Variáveis Regionalizadas, a qual afirma que a variação espacial de um fenômeno é estatisticamente homogênea em uma área, por tentar expressar tendências sugeridas pelos dados (MARCUZZO et al., 2011; MEDEIROS et al., 2017).

\section{ANÁLISE doS EVENTOS EL NIÑo E LA NIÑA E AS ANOMALIAS PLUVIAIS}

Para identificar-se o aquecimento e o resfriamento das águas do oceano pacífico (Figura 2) correspondiam a eventos de maior e menor precipitação na região da bacia do rio Capim, os dados do Índice Oceânico de Niño (ION) foram extraídos do banco de dados do Climate Prediction Center (CPC-NOAA), de 1950 a 2019, contudo, foi realizada a análise dos anos em estudo, 1983-2014. 


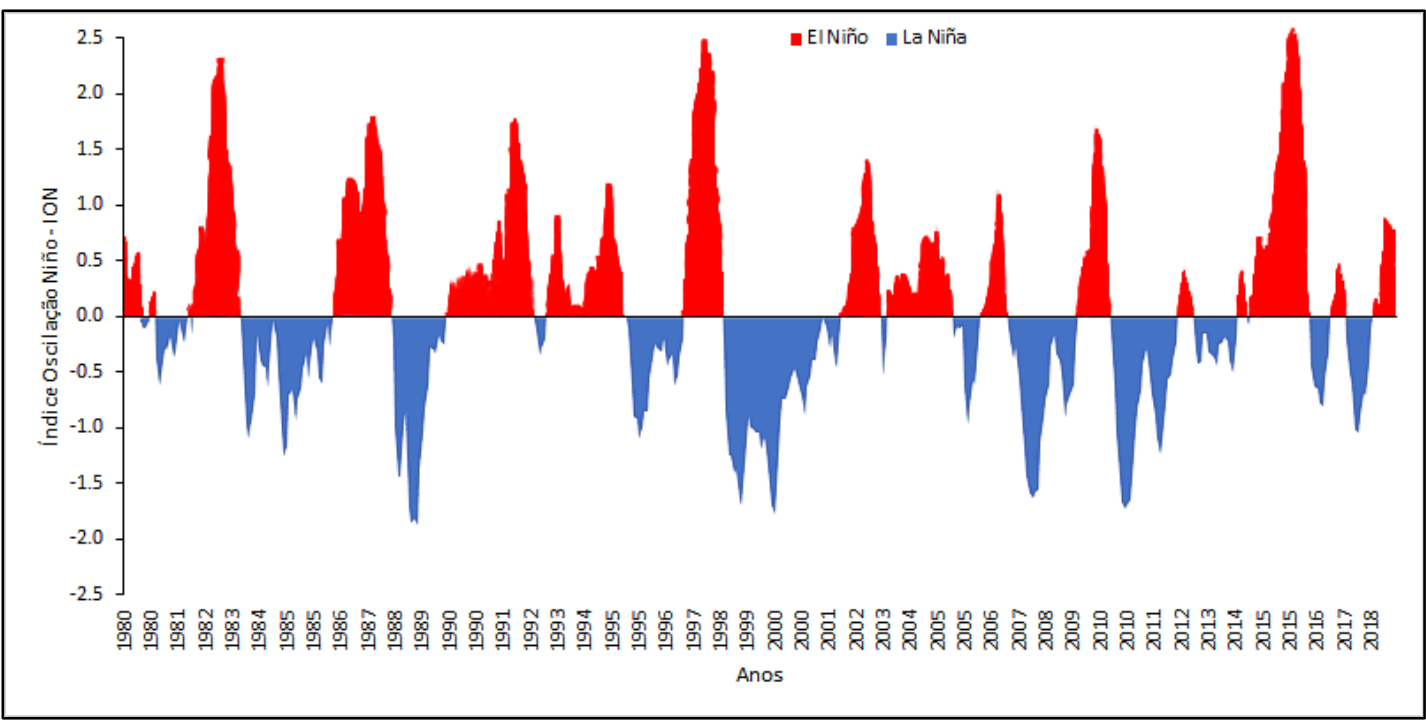

Figura 2 - Índice oceânico de Niño (ION) na região do Niño 3.4.

A National Oceanic and Atmospheric Administration (NOOA, 2017) realiza o monitoramento do Índice Oceânico do $\mathrm{Niño}^{1}$ (ION), o ION é um índice que identifica as anomalias da superfície do mar na região do Niño $\left(5^{\circ} \mathrm{N},-5^{\circ} \mathrm{S}\right)$ e $\left(170^{\circ},-120^{\circ} \mathrm{W}\right)$ no oceano Pacífico. Quando o índice for maior que $+0,5^{\circ} \mathrm{C}$ por no mínimo cinco meses consecutivos determina-se a ocorrência de um El Niño (cor vermelha); quando menor que $-0,5^{\circ} \mathrm{C}$ em cinco meses consecutivos caracteriza-se uma La Niña (cor azul). Além disso, foi extraído produtos de correlação e regressão linear do ENOS com a precipitação, no intuído de confirmar o ENOS como teleconexão com as chuvas na bacia hidrográfica do rio Capim. Os dados de temperatura da superfície do mar (TSM), estão na grade de $\left(0,5^{\circ} \times 0,5^{\circ}\right)$ referente ao Niño 3.4 e os dados de Precipitação são os dados de reanálise do CPC, o GPCC.

A análise da anomalia de precipitação na bacia do rio Capim foi realizada de acordo com (SOUSA et al., 2015 e NORONHA et al., 2016), a partir da precipitação acumulada anual obtida a partir da soma dos meses de cada ano obtidos do GPCC para os anos de 1983 a 2014. A partir desses dados calcularam-se as médias aritméticas e o desvio padrão da precipitação para bacia do rio Capim, para determinar à anomalia de precipitação por meio das equações a seguir.

AP $=$ Pobs $-($ Pmed + DP $)$, se Pobs $>$ Pmed $+D P$

AN $=($ Pmed - DP $)-$ Pobs, se Pobs $<$ Pmed $-D P$

onde: AP é a anomalia positiva; AN é a anomalia negativa; Pobs é a precipitação observada; Pmed é a precipitação media e DP e o desvio padrão do período.

Segundo Sousa et al. (2015), a anomalia positiva (negativa) é definida quando a precipitação é maior (menor) do que o valor da precipitação média menos o desvio padrão do período, respectivamente. Portanto, analisou-se a

\footnotetext{
${ }^{1}$ Disponível em

http://www.cpc.ncep.noaa.gov/products/analysis_monitoring/ensostuff/ensoyears.shtml
} 
bacia do rio Capim, considerando anomalias negativas de precipitação (valores menores que -1), caracterizando o fenômeno El Niño; e anomalias positivas (valores maiores que 1), caracterizando a componente La Niña.

De acordo com Ishihara et al. (2014), por meio da análise da precipitação na região estudada, pode-se avaliar a dinâmica da precipitação no período. Além disso, a partir dos valores médios de precipitação e dos dados de El Niño e La Niña pode-se analisar a relação do aumento ou diminuição da precipitação na região sendo influenciada por esses fenômenos climáticos.

\section{RESULTADOS E DISCUSSÃO}

A Figura 3 apresenta a validação dos dados estimados pelo GPCC com os dados medidos da ANA. É observado que todos os índices estatísticos utilizados foram satisfatórios em validar o GPCC. Nesse sentindo o Coeficiente de correlação (R) obteve o valor de 0.96 , bem como o coeficiente de determinação $\left(R^{2}\right)$ com valor de 0.93. Para os coeficientes de Nash-Sutcliffe (NS) e Willmott obtivemos excelentes valores, com 0.99 para ambos. Já para o coeficiente de $U$ (Theil) foi os dados estimados com os dados observados resultaram em $0.09,0$ que é considerado um ajuste perfeito dos dados. Tais resultados concordam com os resultados encontrados por Serrão et al., (2016) para a bacia hidrográfica do rio Solimões, na validação dos dados da constelação GPM com satélite TRMM. Santos et al., 2014 observou resultados similares pra a bacia hidrográfica do Tapajós. Gonçalves et al., (2016), utilizou esses índices para validar a precipitação estimada pelo polígono de Thiessen e a precipitação estimada pelo TRMM, para a bacia hidrográfica do rio Negro.

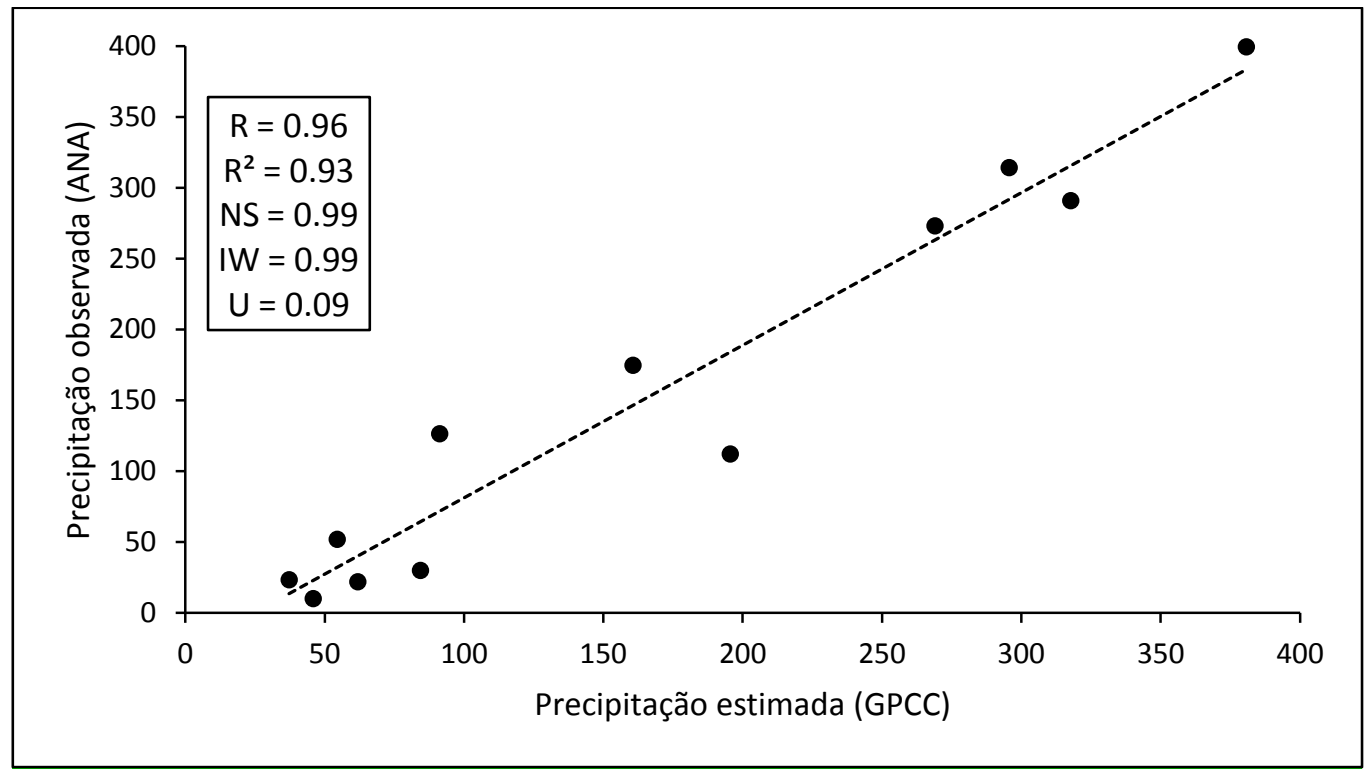

Figura 3 - Validação da precipitação estimada (GPCC) com a precipitação medida (ANA) para bacia do rio Capim.

Durante o período analisado (1983-2014) na região da bacia do rio Capim, a média da precipitação anual foi de $1993 \mathrm{~mm}$, tendo o mês de março como o de maior quantidade precipitada, cerca de $380 \mathrm{~mm}$, já o mês de menor média precipitada foi setembro, em média de $37 \mathrm{~mm}$ (Figura 4). 
Albuquerque et al. (2010) observaram para a região do Capim, uma precipitação anual média de $1837 \mathrm{~mm}$. Já no estudo realizado por Amanajás e Braga (2012), identificaram uma precipitação anual média em torno de 2100 mm. Segundo Moraes et al. (2005), a variação anual da precipitação nessa região está compreendida entre 1800 e 2300 mm.

A precipitação apresentou seu máximo nos meses de dezembro e maio (1619 mm), período mais chuvoso. De acordo com Amanajás e Braga (2012) e Moraes et al. (2015), na região central e norte da Amazônia, o sistema precipitante de maior importância é a ZCIT; e na região sul a ZCAS, atuando concomitantemente no regime pluviométrico da região. A climatologia da Amazônia é também influenciada por Linhas de Instabilidade e outros sistemas de mesoescala (COHEN et al., 1995), além de haver uma forte influência das condições de TSM no Atlântico tropical (Souza et al., 2000).

Observa-se que o trimestre mais chuvoso na bacia do rio Capim é fevereiro a abril com um total médio no trimestre de $994 \mathrm{~mm}$, que representa aproximadamente $50 \%$ do total médio anual. Segundo Amanajás e Braga (2012), a bacia do rio Capim encontra-se localizada na região homogênea 3, a qual é considerada a terceira porção mais chuvosa da região, com total anual em torno de $2100 \mathrm{~mm}$, tendo o trimestre de fevereiro a abril, como o mais chuvoso $(1052,7 \mathrm{~mm})$.

Menezes et al. (2015) discutem que na região do Capim, o período de estiagem sofre antecipação para julho, agosto e setembro indicando a influência dos sistemas sinóticos. Para a região do Capim observa-se a transição sazonal com aumento da precipitação a partir de dezembro e fim da estação chuvosa em maio, com picos em março a precipitação decresce significativamente nos meses posteriores, além de um período menos chuvoso de junho a novembro, isto ocorre porque nesse período a região sofre influência da migração sazonal da ZCIT para o hemisfério norte.

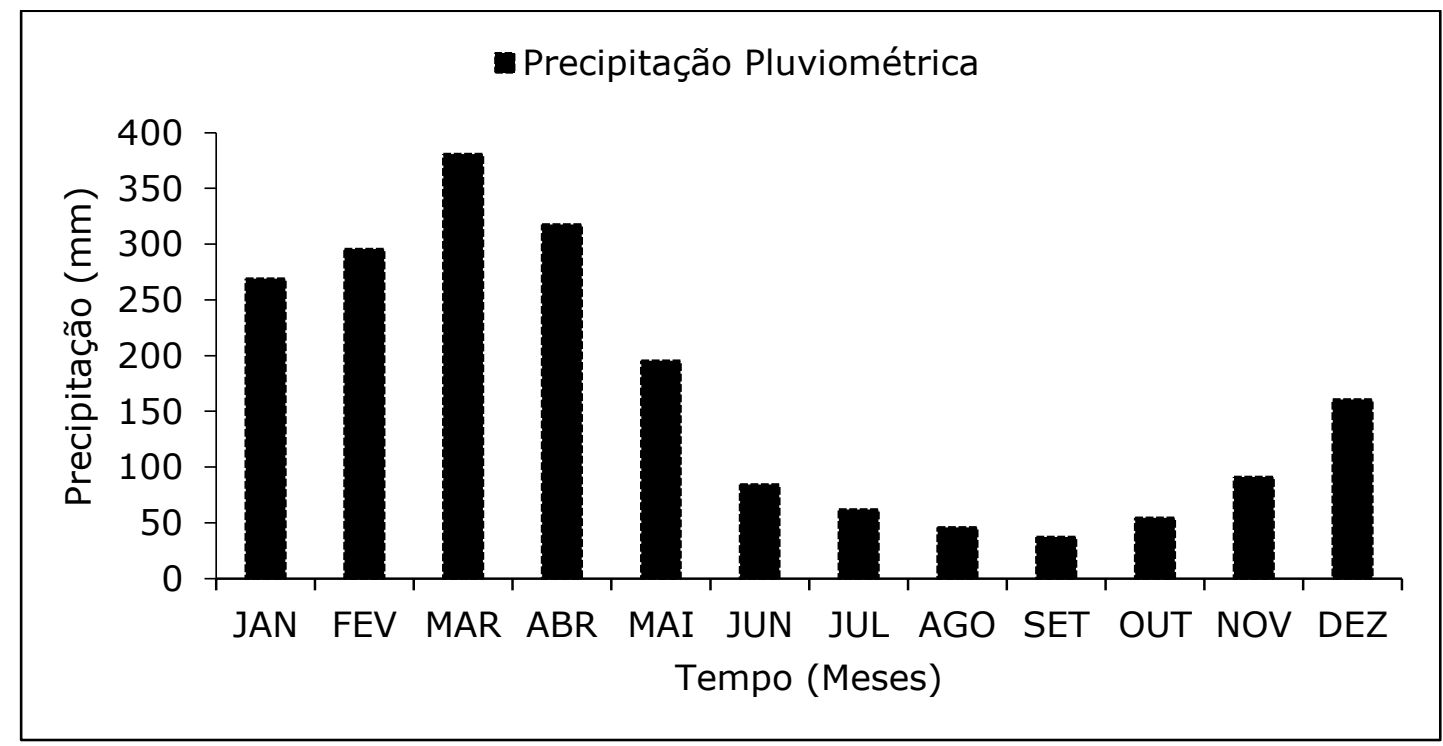

Figura 4 - Média mensal acumulada da precipitação para bacia do rio Capim. 
A teleconexão entre os eventos de ENOS e a precipitação na Amazônia já vem sendo descrita ao longo dos anos, contudo, esta análise no tocante de bacias hidrográficas é de suma importância para o gerenciamento de seus recursos hídricos.

Para Araújo et al. (2013), a variabilidade pluviométrica na região Norte da América do Sul está relacionada com a ocorrência de ENOS e ainda sugerem que tais fenômenos oceano-atmosférico tem impactado o clima regional; e os mesmos reforçam que estas anomalias influenciam mais no período chuvoso, além de não serem os únicos fatores devido a ação antrópica, topografia e TSM oriunda do Atlântico que afeta significativamente no clima da Amazônia (SOUSA et al., 2018).

Tais resultados já haviam sido relatados em diversos estudos (ANGSTRÖM, 1935; Kousky et al., 1984; Barnston e Ropelewski, 1992; Marengo, 1992; Kousky, 1997; Grimm, 2003; Reboita et al., 2010; Merengo et al., 2018).

No âmbito regional, foi feito a correlação entre as anomalias de precipitação e o ION para o ano de 1997/1998, tais anos são marcados por terem sido, até então, o El Niño mais forte da história, superado pelo El Niño 2015/2016. Na figura 5 observamos a correlação negativa entre a precipitação e o índice de Niño. Sendo o coeficiente de Person $(R)$ de - 0,5 e o coeficiente de Determinação $\left(R^{2}\right)$ de 0,2 .

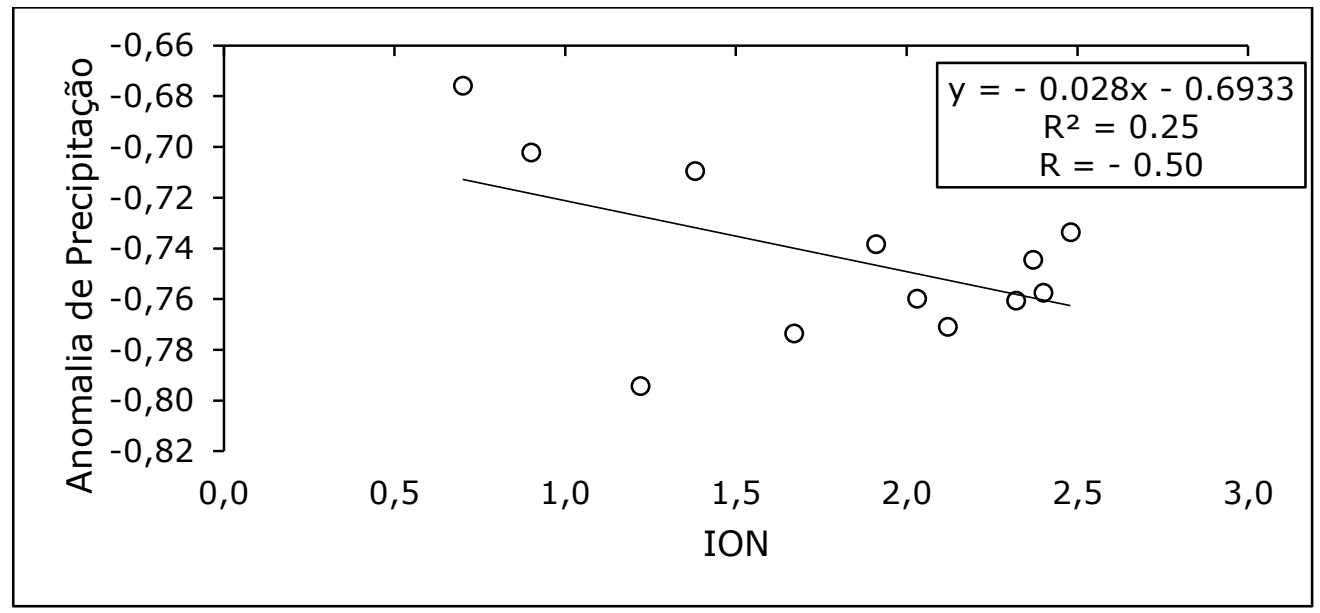

Figura 5 - Regreção linear entre a anomalia de precipitação e o ION para bacia do rio Capim.

Segundo Amanajás e Braga (2012), os mínimos de precipitação para o estado do Pará foram nos anos de 1983, 1992 e 1998, indicando que houve uma redução considerável da precipitação no semestre mais chuvoso, resultados semelhantes aos obtidos para a bacia do rio Capim, a qual teve como anos de menor precipitação 1983, 1987, 1992, 1997 e 1998. De acordo com os autores, esses mínimos observados estão associados aos eventos ENOS e, em menor proporção, aos episódios de Dipolo do Atlântico.

Segundo Albuquerque et al. (2010), nos anos de 1984 a 1986; 1994 a 1996; 1999 a 2000, ocorreram índices de precipitação acima do normal em grande parte do estado do Pará, estes picos de precipitação estão associados ao fenômeno La Niña, que induzida pela ZCIT favorece a ocorrência de precipitação 
acima do normal em grande parte do Estado. Quando se analisa a região da bacia do rio Capim, identifica-se que nos anos de 1984 a 1986; 1994 a 1996; 1999 a 2000, também ocorreram índices pluviométricos acima da média, o que demostra que essa região foi atingida pelo fenômeno La Niña. Já na mesorregião sudeste do estado ocorreu menores índices pluviométricos, pelo retraimento dos episódios de ZCAS.

De acordo com Serrão et al. (2017), a diminuição da precipitação em 2010 foi relacionada com a ocorrência dos sistemas acoplados oceano-atmosfera de grande escala (EI Niño e Dipolo do Atlântico), estes influenciaram os níveis pluviométricos, desintensificando as chuvas. Além disso, nos períodos do verão e outono de 1982/83 também ocorreu a diminuição da precipitação na região amazônica pela manifestação desses fenômenos, os quais diminuem à ocorrência de chuvas na Amazônia.

Os impactos da ENSO no globo são em grande parte lineares, o que significa que a fase quente (El Niño) é geralmente associada às anomalias negativas de precipitação e a fase fria (La Niña) pode ser visualizado invertendo o sinal das anomalias (Ropelewski e Halpert, 1996). Eventos de El Niño (La Niña) concomitante com anomalias de TSM no atlântico tropical, intensificam (diminuem) a precipitação sobre a porção central-leste da Amazônia e nordeste Brasileiro (MARENGO et al., 2018)

A partir da análise da anomalia de precipitação na bacia do rio Capim dos anos 1983-2014 (Figura 6), identificou-se que normalmente os anos abaixo (acima) do desvio padrão foram anos de ocorrência do fenômeno El Niño (La Niña) de acordo com os dados da National Oceanic and Atmospheric Administration (NOAA, 2018), a qual realiza o monitoramento do ION.

Os anos de 1984, 1985, 1988, 1989, 1996, 2000, 2008, 2009 e 2011 houveram as maiores ocorrências de precipitação (média de 2331,6 mm/ano), já os anos de 1983, 1987, 1992, 1997, 1998, 2010 foram os menos chuvosos (média de 1607,2 mm/ano), estes períodos coincidem respectivamente com as influências dos fenômenos de La Niña e El Niño na região Amazônica. Essas médias são diferentes da média considerando todos os anos estudados (1993 $\mathrm{mm} / a n o)$. Tais precipitações intermediárias confirma a tendência de maior precipitação para os anos de La Niña e tendência de menor precipitação para os anos de El Niño (GONÇALVES et al., 2016). 


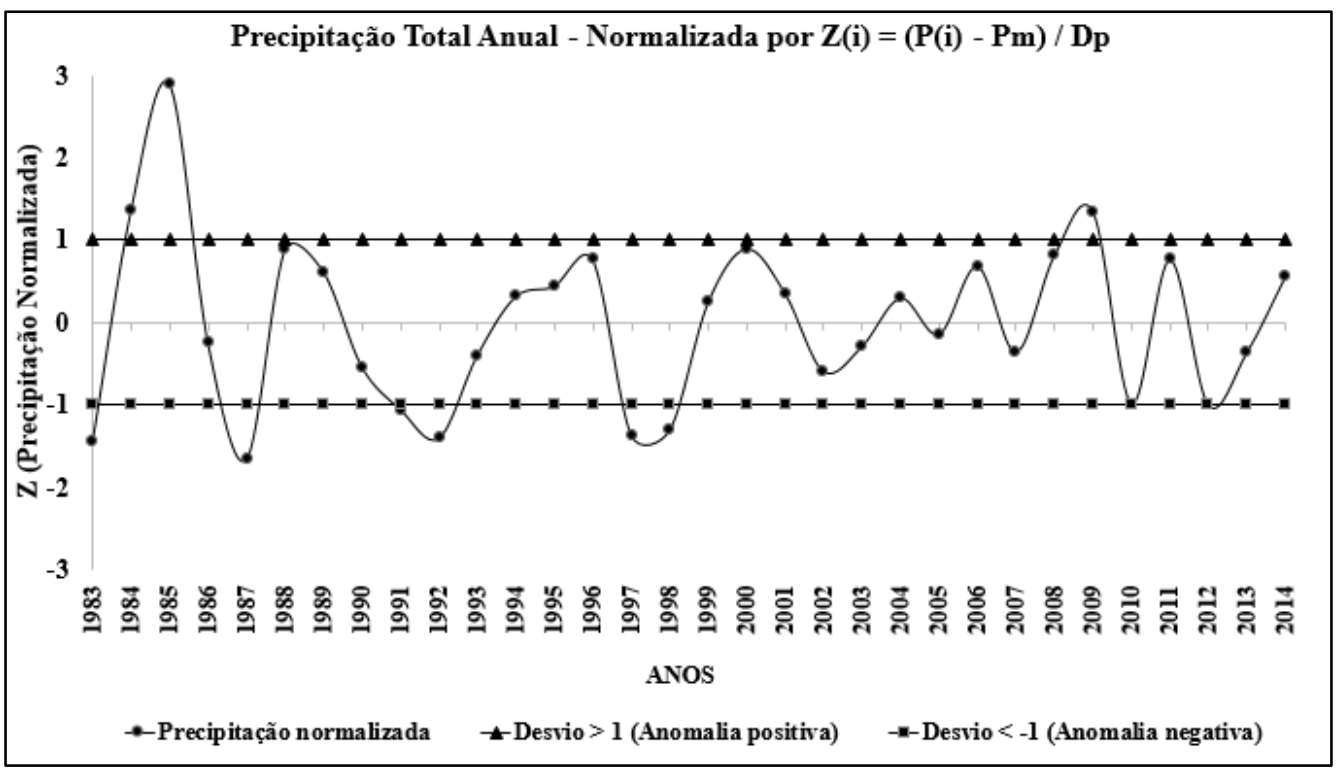

Figura 6 - Anomalia de precipitação para bacia do rio Capim.

Tais anos são anomalamente negativos e positivos de precipitação para a região amazônica, especialmente os anos de 1998, 2005 e 2010 concordando com os resultados de Serrão, et al., (2015).

Segundo Marengo e Espinoza (2015), o ano de 2005 apresentou precipitação abaixo do normal para a região amazônica, ocasionando seca em diversas áreas da região, devido ao aquecimento das águas do oceano Pacífico. Porém, o ano de 2005 não apresentou uma precipitação muito abaixo da média para a região do Capim, pois, conforme Araújo e Ponte (2016) a região da bacia do Capim não está localizada dentro da área afetada pelo fenômeno, o qual foi concentrado na porção sudoeste da bacia Amazônica.

Percebe-se que a precipitação na bacia do rio Capim foi mais afetada no ano de 2010 do que em 2005. Segundo Lewis et al. (2011), uma das possíveis explicações para esse efeito é o fato das anomalias pluviais na região amazônica terem reduzido a chuva em 57\% (2010) em comparação a 37\% (2005). Estas anomalias pluviométricas foram causadas pela teleconexão entre um evento de El Niño e o Dipolo do Atlântico positivo, outrossim, as anomalias de precipitação foram ainda mais intensas do outono a primavera, ou seja, diminuindo a a precipitação na estação chuvosa e coincidindo com o período seco da região (junho a setembro), essas anomalias de chuva reduziram o nível de cota fluvial dos maiores rios da Amazônia e foi considerada a seca do século (Marengo et al., 2005; Marengo et al., 2011; Serrão et al., 2015).

Os resultados para a espacialização da precipitação média anual acumulada (Figura 7) mostraram que a bacia hidrográfica do rio Capim tem a região menos chuvosa a sudeste da bacia, apresentando valores de precipitação anual em torno de $1.700 \mathrm{~mm}$; a região com registros mais elevados de precipitação situa-se na parte norte da bacia com valores em torno de 2.400 $\mathrm{mm}$. Estes resultados estão de acordo com os obtidos por Ishihara et al. (2014), Santos et al. (2014b), Menezes e Fernandes (2016) e Gomes et al. (2018). 


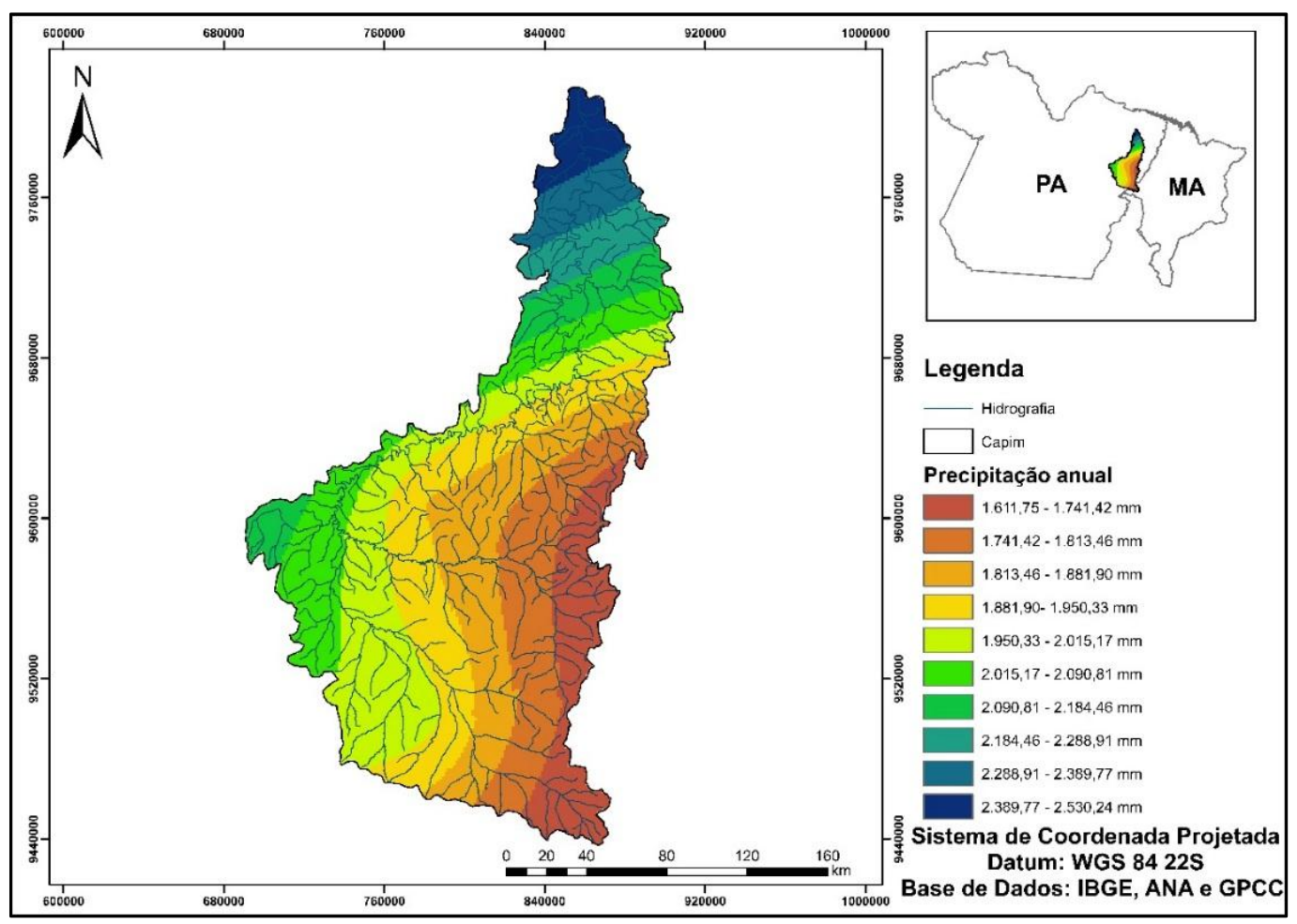

Figura 7 - Distribuição espacial da média anual acumulada da precipitação.

A sub-bacia do Baixo Capim apresenta os maiores volumes de chuva da região, superior a $2300 \mathrm{~mm} / a n o$, caracterizando esta região como a de maior potencial hídrico da bacia; a região com menores valores pluviométricos está localizada no extremo sudeste da bacia, com aproximadamente 1.600 mm/ano.

Na Figura 8 ilustra a distribuição pluviométrica mensal, onde os meses de dezembro a maio são marcados como o período chuvoso (135 mm - $450 \mathrm{~mm}$ ), sendo este montante pluvial favorecido pelo deslocamento meridional da ZCIT ao sul (COUTINHO et al., 2018). O período de estiagem é caracterizado nos meses de junho a novembro (0 mm - $135 \mathrm{~mm})$, no qual o sistema meteorológico atuante desloca-se na direção norte diminuindo as chuvas na região. O mês com menor volume de precipitação é agosto com precipitações mais baixas ocorrendo na parte centro-sul da bacia.

A partir da figura abaixo consegue-se identificar o começo da diminuição da precipitação no mês de maio, o qual corresponde ao fim do período chuvoso. O mês de setembro é o de menor precipitação para região estudada, a média da precipitação nesse mês foi de aproximadamente $37 \mathrm{~mm}$.

A diminuição da precipitação é mais perceptível analisando-se a porção sudeste da bacia. De forma geral as chuvas se concentram na porção norte tendo no mês de maio alcançado o máximo de aproximadamente $300 \mathrm{~mm}$ (junho-agosto), correspondendo ao período de menor taxa de precipitação na região.

Como todos os meses, apresentam uma redução da precipitação no sentido norte-sul da bacia, chegando a aproximadamente $30 \mathrm{~mm}$ na região sudeste da bacia. Já no mês de dezembro inicia-se a estação chuvosa, a bacia 
do rio Capim entra na fase de recuperação hídrica, onde observa-se que os totais pluviométricos variam de $140 \mathrm{~mm}$, ao sul da bacia, com os valores aumentando até 190 ao norte da bacia.

Os resultados obtidos pela análise da espacialização da precipitação, demonstram que as sub-bacias Ararandeua e Surubiju representam a zona de maior variação da precipitação ao longo do ano.

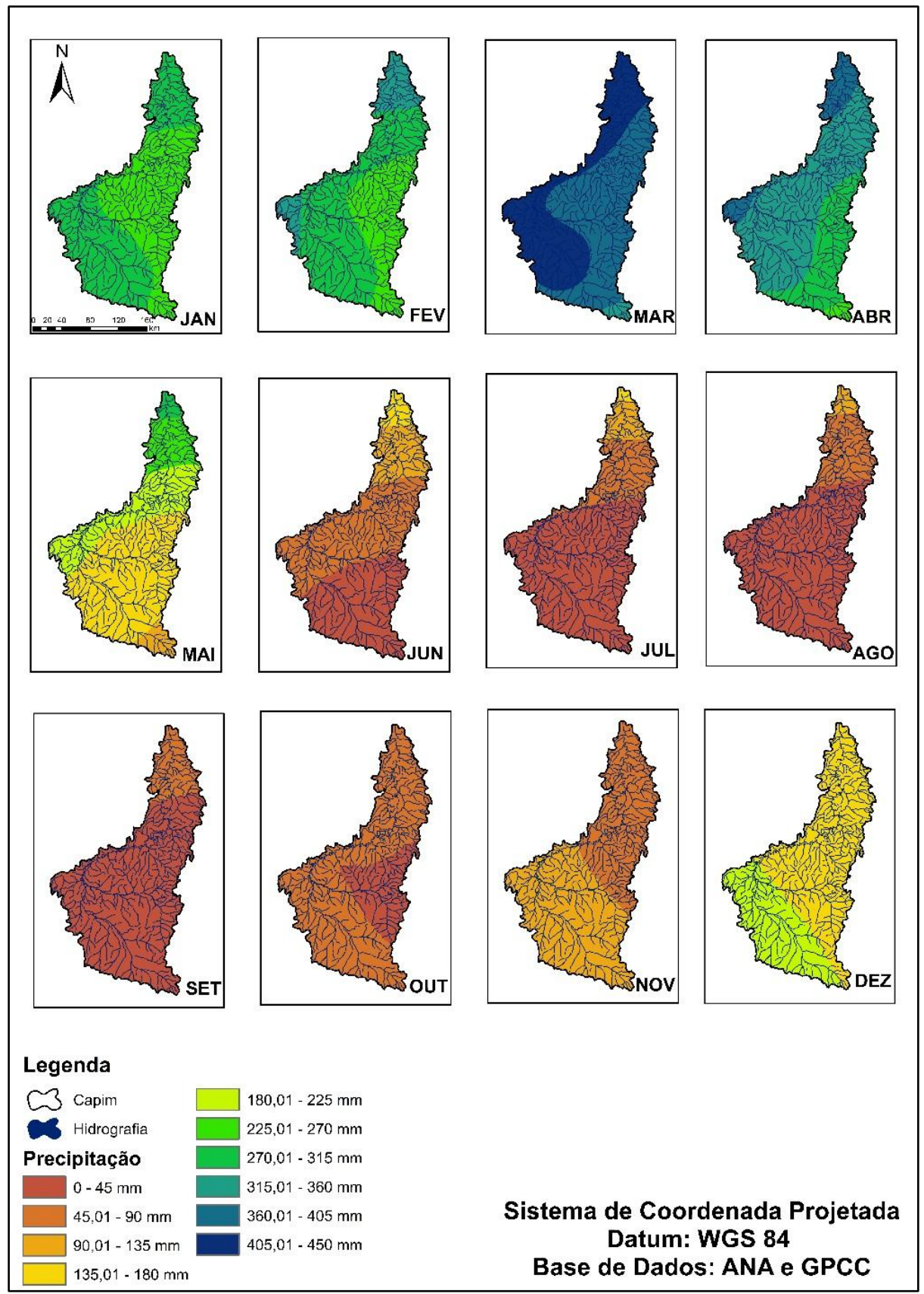

Figura 8 - Distribuição espacial da precipitação média mensal. 


\section{CONCLUSÃO}

Por meio da espacialização da precipitação na bacia do rio Capim foram observados maiores valores de precipitação para a região do baixo-médio Capim e menores valores para o alto Capim, caracterizando-se estas regiões como as de maior potencial hídrico e menor potencial hídrico da bacia, respectivamente. Tal variabilidade está de acordo com os sistemas atmosféricos que atuam na bacia, bem como a variabilidade mensal configura um período mais chuvoso de dezembro a maio e outro menos chuvoso de junho a novembro. Essa precipitação é resultante dos diversos sistemas atmosféricos que atuam na Amazônia.

A validação dos dados do GPCC foi de suma importância para sabermos se realmente pode-se utilizar os dados do GPCC como substituto para os dados medidos das agências Brasileiras, nesse sentindo, todos os índices estatísticos utilizados obtiveram valores considerados excelentes. Com a correlação das anomalias de precipitação e o ION, nos permitiu confirmar a hipótese que os eventos de ENOS modulam negativamente a precipitação na bacia hidrográfica do rio Capim.

Observou-se que o regime de chuvas sofre influência dos fatores regionais associados ao fenômeno ENOS. A região da bacia do rio Capim foi afetada pelo evento El Niño 1987 que apresentou maior intensidade do que os eventos de 1997 e 1998. A bacia também sofreu a influência da La Niña 1984/85 com o aumento da precipitação observada. Desta forma, o monitoramento deste fenômeno é fundamental para a mitigação dos impactos ambientais ocasionados na região do Capim.

\section{REFERÊNCIAS BIBLIOGRÁFICAS}

ANGSTRÖM, A. Teleconnections of climate changes in present time. Geography Annals, v. 17, p 242- 258, 1935.

ALBUQUERQUE, M. F.; SOUZA, E. B.; OliVEIRA, M. C. F.; SOUZA JÚNIOR, J. A. Precipitação nas mesorregiões do estado do Pará: climatologia, variabilidade e tendências nas últimas décadas (1978-2008). Revista Brasileira de Climatologia, v. 6, p. 151-168. 2010.

AMANAJÁS, J. C.; BRAGA, C. C. Padrões Espaço-temporal Pluviométricos na Amazônia Oriental Utilizando Análise Multivariada. Revista Brasileira de Meteorologia, v. 27, n. 4, p. 423 - 434, 2012.

ANDREOLI, R. V.; KAYANO, M. T. ENSO-related rainfall anomalies in South America and associated circulation features during warm and cold pacific decadal oscillation regimes. International Journal of Climatology, v. 25, p. 20172030, 2005.

ARAUJO, R. C.; PONTE, M. X. Efeitos do desmatamento em larga-escala na hidrologia da bacia do Uraim, Amazônia. Revista Brasileira de Geografia Física, v. 9, n. 7, p. 2390-2404, 2016.

ARAÚJO, R. G.; ANDREOLI, R. V.; CANDIDO, L. A.; KAYANO, M. T.; SOUZA, R. A. F. A influência do evento El Niño - Oscilação Sul e Atlântico Equatorial na precipitação sobre regiões norte e nordeste da América do Sul. Revista Acta Amazônia. v. 43, n. 04, p. 469 - 480, 2013. 
ÁVILA, P. L. R.; SOUZA, E. B.; PINHeIRO, A. M. FIGUEIRA, W. S. Análise da precipitação sazonal simulada utilizando o regcm4 sobre o estado do Pará em anos de extremos climáticos. Revista Brasileira de Climatologia, v. 14, p. 247263, 2014.

BARNSTON, A.G.; C. F. ROPELEWSKI. Prediction of ENSO episodes using canonical correlation analysis. Jornal of Climate, v. 5, p. 1316-1345, 1992.

BERTONI, J.C.; TUCCI, C.E.M. Precipitação. In: TUCCI, C.E.M.; SILVEIRA, A. L. L. (EDS.). Hidrologia: ciência e aplicação. Porto Alegre: ABRH, 2009.

BRAGA, A. P. Estudo da variabilidade interanual associada ao El Niño e La Niña sobre o regime hidropluviométrico da Bacia do Rio Guamá-Capim. Monografia de especialização em Meteorologia Tropical. Universidade Federal do Pará, Belém, 2001.

CARAM, R. O. Mudanças no uso e cobertura do solo e resposta hidrológica da bacia do rio Piraricaba. 2010. 141 f. Tese (Doutorado em Ciências) - Escola Superior de Agricultura "Luiz de Queiroz", Universidade de São Paulo, Piracicaba, 2010.

COHEN, J.C.P.; SILVA DIAS, M.A.F.; NOBRE, C.A. Environmental Conditions Associated with Amazonian Squall Lines: A case Study. Monthly Weather Review, v. 123, n. 11, p. 3163-3174. 1995.

COUTINHO, E. C.; ROCHA, E. J. P.; LIMA, A. M. M.; RIBEIRO, H. M. C.; GUTIERREZ, L. A. C. L.; BARBOSA, A. J. S.; PAES, G. K. A. A.; BISPO, C. J. C.; TAVARES, P. A. Variabilidade climática da precipitação na bacia amazônica brasileira entre 1982 e 2012. Revista Brasileira de Climatologia. v. 22, p.476 500, 2018.

Environmental Systems Research Institute - ESRI. ArcGis for students. Disponivel em: <https://www.esri.com/en-us/arcgis/products/arcgis-forstudent-use>, Acesso em: 20 jan 2018.

GOMES, D. J. C.; LIMA, A. M. M.; SILVA JUNIOR, J. A. SERRÃO, E. A. O. Impactos do uso das mudanças do uso da terra e dos eventos climáticos extremos em sistemas hidrológicos da Amazônia oriental - Bacia do rio Capim. Revista Geográfica Acadêmica, v. 12, n. 2, p. 153 - 172, 2018.

GOMES, N. V. Estudo comparativo da distribuição espaço-temporal da precipitação na Amazônia oriental. Dissertação de Mestrado em Ciências Ambientais. UFPA, 2008.

GONÇALVES, L. J. M.; ROCHA, E. J. P.; LIMA, A. M. M.; SERRÃO, E. A. O.; SANTOS, C. A. Variabilidade da precipitação e a produção de arroz na bacia hidrográfica do rio Branco. Ambiência, v. 12, n. 4, p. 835-855, set.-dez. 2016.

GRIMM, A. M. The El Niño impact on the summer monsoon in Brazil: regional processes versus remote influences. Journal of Climate, v. 16, p. 263-280. 2003.

GUILHON, L. G. F.; ROCHA, V. R. Comparação de Métodos de Previsão de Vazões Naturais Afluentes a Aproveitamentos Hidroelétricos. Revista Brasileira de Recursos Hídricos. v.12, n. 3, p. 13-20. 2007. 
HOFFMANN, E. L.; DALLACORT, R.; YAMASHITA, O. M.; BARBIERI, J. D. Variabilidade das chuvas no sudeste da Amazônia paraense, Brasil. Revista Brasileira de Geografia Física. v. 11, n. 04, p. 1251 - 1263, p. 2018.

ISHIHARA, J. H.; FERNANDES, L. L.; DUARTE, A. A. A. M.; DUARTE, A. R. C. L. M.; PONTE, M. X.; LOUREIRO, G. E. Quantitative and Spatial Assessment of Precipitation in the Brazilian Amazon (Legal Amazon) - (1978 to 2007). Revista Brasileira De Recursos Hídricos, Porto Alegre, vol. 19, n. 1, p. 29 - 39, 2014.

KOUSKY, V. E.; KAYANO, M. T.; CAVALCANTI, I. F. A. The Southern Oscillation: Oceanic-atmospheric circulation changes and related rainfall anomalies. Tellus, v. 36 A, p. $490-504.1984$.

KOUSKY, V. E. Warm (El Nino) episode conditions return to the tropical Pacific. Mariners Weather Log, v. 41, n. 1, p. 4-7, 1997.

LEWIS, S. L.; BRANDO, P. M.; PHILLIPS, O. L.; VAN DER HEIJDEN, G. M. F.; NEPSTAD, D. The 2010 Amazon drought. Science, v. 331, n. 6017, p. 311-554, 2011.

LIMA, A. M. M. O planejamento estratégico e a gestão da oferta hídrica baseados no estudo da paisagem, na bacia do rio Capim - PA. 2007. 313 f. Tese (Doutorado em Ciências: Desenvolvimento Socioambiental) - Universidade Federal do Pará, Belém, 2007.

LIMA, A. M. M.; PONTE, M. X. Dinâmica da Paisagem da Bacia do Rio Capim-PA. Revista Brasileira de Geografia Física, v. 1, p. 127-142, 2012.

LUNDGREN, W. J. C.; SOUZA, I. F.; LUNDGREN, G. A. Krigagem na construção de mapa pluviométrico do Estado de Sergipe. Revista Brasileira de Geografia Física, v. 10, n. 1, p. 13-22, 2017.

MARCUZZO, F. F. N.; ANDRADE, L. R.; MELO, D. C. R. Métodos de Interpolação Matemática no Mapeamento de Chuvas do Estado do Mato Grosso. Revista Brasileira de Geografia Física, v. 4, p. 793-804, 2011.

MARCUZZO, F. F. N.; ROMERO, V. Influência do El Niño e La Niña na precipitação máxima diária do estado de Goiás. Revista Brasileira de Meteorologia, v. 28, n. 4, 429 - 440, 2013.

MARENGO, J. A. Interanual variability of surface climate in the amazon basin. International journal of climatology, v. 12, p. 853-863, 1992.

MARENGO J. A.; NOBRE. C.; TOMASELLA, J.; OYAMA, M.; OlIVEIRA, G. S.; OLIVEIRA, R.; CAMARGO, H.; ALVES, L. M.; BROWN, I. F. The drought of Amazônia in 2005. Journal of Climate, n. 21, p. 495-516, 2008.

MARENGO, J. A.; TOMASELLA, J.; ALVES, L. M.; SOARES. W. R.; RODRIGUEZ, D. A. The drought of 2010 in the context of historical droughts in the Amazon region. Geophysical Research Letters, v. 38, p. 1-5, 2011b.

MARENGO, J.A.; ESPINOZA, J.C. Extreme seasonal droughts and floods in Amazonia: causes, trends and impacts. International Journal of Climatology, $v$. 36. 2015.

MARENGo, J. A.; AlVes, L. M.; AlvalA, R. C. S.; CUNHA, A. P.; BRITO, S.; MORAES, O. L. L. Climatic characteristics of the 2010-2016 drought in the 
semiarid Northeast Brazil region. Anais da Academia Brasileira de Ciências, v. 90, n.1, p. 1973-1985, 2018.

MEDEIROS, F. J.; LUCIO, P. S.; SILVA, H. J. F. Análise de Métodos de Krigagem na Estimativa da Precipitação no Estado do Rio Grande do Norte. Revista Brasileira de Geografia Física, v. 10, n. 5, p. 1668-1676, 2017.

MENEZES, F. P.; FERNANDES, L. L. Análise de tendência e variabilidade da precipitação no estado do Pará. Enciclopédia biosfera, v.13, n. 24, p. 15801591. 2016.

MENEZES, F. P.; FERNANDES, L. L.; ROCHA, E. J. P. O uso da estatística para regionalização da precipitação no estado do Pará, Brasil. Revista Brasileira de Climatologia, v. 16, jan.-jul., p. 64-71. 2015.

MORAES, B. C.; SODRÉ, G. R. C.; SOUZA, E. B.; FERREIRA, D. B. S.; OLIVEIRA, J. V. Sensoriamento remoto como ferramenta para a determinação da estação chuvosa na Amazônia. Revista Brasileira de Geografia Física, v. 8 n. 5, p. 13741382. 2015.

NASCIMENTO, T. S.; SARAIVA, J. M.; SENNA, R.; AGUIAR, F. E. Preenchimento de falhas em banco de dados pluviométricos com base em dados do CPC (Climate Prediction Center): Estudo de caso do rio Solimões - Amazonas. Revista Brasileira de Climatologia, v. 07, n. 06, p. 143 - 158, 2009.

National Oceanic and Atmospheric Administration - NOAA. Cold \& warm episodes by season. Disponível em: <http://www.cpc.ncep.noaa.gov/products/analysis_monitoring/ensostuff/ensoye ars.shtml>. Acesso em: 10 jan. 2018.

Climate Prediction Center - CPC/NOAA. Global ENSO Temperature \& Precipitation Regressions Information. Disponível em: <https://origin.cpc.ncep.noaa.gov/products/precip/CWlink/ENSO/regressions/re adme.shtml>. Acesso em 30 mar 2019.

NASH, J. E.; SUTCLIFFE, J. V. River flow forecasting through conceptual models: a discussion of principles. Journal of Hydrology, v. 10, p. 282-290. 1970.

NORONHA, G. C.; DA HORA, M. A. G. M.; SILVA, L. P. Análise do Índice de Anomalia de Chuva para a Microbacia de Santa Maria/Cambiocó, RJ. Revista Brasileira de Meteorologia, v. 31, n. 1, p. 74-81, 2016.

REBOITA, M.; GAN, M.; ROCHA, R.; AMBRIZZI, T. Regimes de precipitação na América do Sul: uma revisão bibliográfica. Revista Brasileira de Meteorologia, $v$. 25, p. 185-204, 2010.

ROPELEWSKI, C. F.; M. S. HALPERT. Quantifying Southern Oscillationprecipitation relationships. Jornal of Climate, v. 9, p. 1043-1059, 1996.

SANTOS, C. A.; SERRÃO, E. A. O.; GONÇALVES, L. J. M.; WANZELER, R. T. S.; LIMA, A. M. M. Zoneamento da distribuição da precipitação pluviométrica na bacia hidrográfica do rio tapajós. Enciclopédia Biosfera, v. 10, n. 18, p. 30923106. 2014.

SANTOS, S. R. Q.; BRAGA, C. C. ; CAMPOS, T. L. O. B.; BRITO, J. I. B.; SANTOS, A. P. P. Variabilidade da Precipitação no Estado do Pará por meio de Análise em Componentes Principais. Revista Brasileira de Geografia Física, v. 7, n. 3, p. 615-627. 2014. 
SCHNEIDER, U.; ZIESE, M.; MEYER-CHRISTOFFER, A.; FINGER, P.; RUSTEMEIER, E.; BECKER, A. The new portfolio of global precipitation data products of the Global Precipitation Climatology Centre suitable to assess and quantify the global water cycle and resources. Proceedings of the International Association of Hydrological Sciences, v. 374, p. 29-34. 2016.

SCHNEIDER, U.; FINGER, P.; MEYER-CHRISTOFFER, A.; RUSTEMEIER, E.; ZIESE, M.; BECKER, A. Evaluating the Hydrological Cycle over Land Using the Newly-Corrected Precipitation Climatology from the Global Precipitation Climatology Centre (GPCC). Atmosphere, v. 8, n. 3, p. 1-17. 2017.

SERRÃO, E. A. O.; SANTOS, C. A.; WANZELER, R. T. S.; GONÇALVES, L. J. M.; LIMA, A. M. M. Avaliação da seca de 2005 e 2010 na Amazônia: uma análise a bacia hidrográfica do rio Solimões. Revista Geográfica Acadêmica, v. 9, n. 2, p. 5-20, 2015.

SERRÃO, E. A. O.; WANZELER, R. T. S.; SANTOS, C. A.; GONÇALVES, L. J. M.; LIMA, A. M. M.; ROCHA, E. J. P. Avaliação Estatística entre as Estimativas de Precipitação da Constelação GPM com TRMM: Uma Análise a Bacia Hidrográfica do Rio Solimões. Revista Brasileira de Climatologia, v. 18, p. 256-275, 2016.

SERRÃO, E. A. O.; LIMA, A. M. M.; SOUSA, F. A. S.; FERREIRA, T. R.; SANTOS, C. A.; SILVA JÚNIOR, J. A. Distribuição espacial de intensidade pluviométrica na calha do rio Solimões: estudo de caso a seca de 2010 na Amazônia. Acta Geográfica, Boa Vista, v. 11, n. 2, p. 1-16. 2017.

SILVA, M. K. A. Modelagem hidrológica aplicada ao estudo da vazão da bacia hidrográfica do rio Araguari - MG, a partir das mudanças do uso da terra e cobertura vegetal nativa. 2014. $196 \mathrm{f}$. Tese (Doutorado em Geografia) Instituto de Geografia, Universidade Federal de Uberlândia, Uberlândia - MG, 2014.

SOUSA, A. C.; CANDIDO, L. A.; ANDREOLI, R. V. Variabilidade interanual da precipitação e fluxo de umidade sobre a Amazônia usando o QTCM. Revista Brasileira de Meteorologia, v. 33, n. 01, p. 41 - 56, 2018.

SOUSA, A. M. L.; ROCHA, E. J. P.; VITORINO, M. I.; SOUZA, P. J. O. P.; BOTELHO, M. N. Variabilidade espaço-temporal da precipitação na Amazônia durante eventos ENOS. Revista Brasileira de Geografia Física, v. 8, p. 15-29, 2015.

SOUZA, E. B.; KAYANO, M. T.; TOTA, J.; PEZZI, L.; FISCH, G.; NOBRE, C. Sobre a Influência do El Niño, La Niña e Padrão De Dipolo do Atlântico na precipitação da amazônia durante 1960-1998. Acta amazônica, v. 30, n. 2, p. 305-318. 2000.

SOUZA, E. B.; LOPES, M. N.G.; DA ROCHA, E. J.P.; SOUZA, J. R. S.; DA CUNHA, A. C.; SILVA, R. R.; FERREIRA, D. B.S.; SANTOS, D. M.; DO CARMO, A. M.C. et al. Precipitação sazonal sobre a Amazônia Oriental no período chuvoso: observações e simulações regionais com o REGCM3. Revista Brasileira de Meteorologia, v. 24, n. 2, p. 111-124, 2009.

SOUZA, N. S.; SOUZA, W. J.; CARDOSO, J. M. S. Caracterização hidrológica e influência da cobertura do solo nos parâmetros de vazão do Rio das Fêmeas. Engenharia Sanitária e Ambiental, v. 22, n. 3, p. 453-462, 2017. 
SOUZA, P. F. S.; SOUZA, E. B.; QUEIROZ, J. C. B.; SILVA JUNIOR, J. A. Impactos dos anos climáticos extremos no rendimento da lavoura temporária de mandioca na região rural da metrópole de Belém - Pará. Revista Brasileira de Climatologia, v. 21, p. 94-112, 2017.

SPENCER, D. E. Developing a Bayesian vector autoregressive forecasting model. International Journal of Forecasting. v. 9, p. 407-421. 1993.

WANDERLEY, H. S. et al. Variabilidade espacial e preenchimento de falhas de dados pluviométricos para o Estado de Alagoas. Revista Brasileira de Meteorologia, v. 27, n. 3, p. 347- 354, 2012 